\title{
Design, development, and validation of an in-situ biosensor array for metabolite monitoring of cell cultures
}

\author{
Cristina Boero ${ }^{\mathrm{a}, *}$, Maria Antonietta Casulli ${ }^{\mathrm{a}}$, Jacopo Olivo ${ }^{\mathrm{a}}$, Lorenzo Foglia ${ }^{\mathrm{a}}$, Eric Orso ${ }^{\mathrm{b}}$, \\ Marco Mazza $^{\mathrm{b}}$, Sandro Carrara ${ }^{\mathrm{a}}$, Giovanni De Micheli ${ }^{\mathrm{a}}$ \\ a Laboratory of Integrated Systems, EPFL, Lausanne 1015, Switzerland \\ ${ }^{\mathrm{b}}$ Department of Electronics Engineering, EIA, Fribourg 1705, Switzerland
}

\section{A R T I C L E I N F O}

\section{Article history:}

Received 6 February 2014

Received in revised form

7 May 2014

Accepted 13 May 2014

Available online 22 May 2014

\section{Keywords:}

Electrochemical biosensor

Metabolite

Cell culture

Carbon nanotubes

Oxidase

Glucose deprivation

\begin{abstract}
A B S T R A C T
Conventional pharmaceutical processes involving cell culture growth are generally taken under control with expensive and long laboratory tests performed by direct sampling to evaluate quality. This traditional and well-established approach is just partially adequate in providing information about cell state. Electrochemical enzyme-based biosensors offer several advantages towards this application. In particular, they lend themselves to miniaturization and integration with cheap electronics. In the present work we go through the design, the development, and the validation of a self-contained device for the on-line measurement of metabolites in cell culture media. We microfabricated a sensing platform by using thin film technologies. We exploited electrodeposition to precisely immobilize carbon nanotubes and enzymes on miniaturized working electrodes. We designed and realized the electronics to perform the electrochemical measurements and an Android application to display the measurements on smartphones and tablets. In cell culture media glucose biosensor shows a sensitivity of $4.7 \pm$ $1.3 \mathrm{nA} \mathrm{mM}^{-1} \mathrm{~mm}^{-2}$ and a detection limit of $1.4 \mathrm{mM}(\mathrm{S} / \mathrm{N}=3 \sigma)$, while for lactate biosensor the sensitivity is $12.2 \pm 3.8 \mathrm{nA} \mathrm{mM}{ }^{-1} \mathrm{~mm}^{-2}$ and the detection limit is $0.3 \mathrm{mM}$. The whole system was then validated by monitoring U937 cell line over $88 \mathrm{~h}$. Metabolic trends were fully congruent with cell density and viability. This self-contained device is a promising tool to provide more detailed information on cell metabolism that are unprecedented in cell biology.
\end{abstract}

(c) 2014 Elsevier B.V. All rights reserved.

\section{Introduction}

Pharmaceutical research, therapies development, diagnostics, regenerative medicine are some of the fields demanding smart, high-sensitive, and non-invasive platforms to better control biological mechanisms. Antibiotics, vaccines, and therapeutic proteins, for example, are increasingly produced by microorganisms, such as bacteria, yeasts, and animal cell lines. Conventional pharmaceutical processes involving cell culture growth are generally taken under control by expensive and long laboratory testing performed by direct sampling to evaluate the quality. This traditional and well-established approach is just partially adequate in providing information about cell state (Vojinović et al., 2006). As an example, drug efficiency is typically tested in vitro, prior to run clinical trials in vivo. The knowledge of the complex cascade of reactions involved in cell mechanisms and variation in cell

\footnotetext{
*Corresponding author. Tel.: +4121 6930919; fax: +41216930909.

E-mail address: cristina.boero@epfl.ch (C. Boero).
}

metabolism can help to infer the potential efficacy of drugs and therapies, at least in vitro. Therefore, the technologies developed in the last decade offer significant opportunities for improving pharmaceutical manufacturing, quality assurance, and process optimization through process control and analysis (FDA, 2004).

Electrochemical enzyme-based biosensors have been largely used in several applications because of their many advantages (Kimmel et al., 2012; Jacobs et al., 2010; D'Orazio, 2011). Since they are based on electrochemical principles, they require simple and cheap electronics to apply slow signals to the electrochemical cell and to readout the current. They provide real-time information and they do not need any regeneration method of the biological recognition element, unlike antibody and DNA strain-based sensors. In particular, oxidase-based biosensors show high versatility, since the immobilization strategy used for one type of enzyme can be also employed for several others and the readout by-product, the hydrogen peroxide $\left(\mathrm{H}_{2} \mathrm{O}_{2}\right)$, is common for all these redox reactions. Therefore, the real challenges from the technological point-of-view are at the integration level: the development of a self-contained device, the assembling of the biolayer with the electronics, the miniaturization of the 
system, the minimization of the noise in the output signal, which is typically very weak, and the low power consumption of these devices (Temiz et al., 2011; Carrara et al., 2011). The detection of $\mathrm{H}_{2} \mathrm{O}_{2}$ can be electrochemically performed on pure metal, such as gold or platinum, on metal alloys, or on carbon-based materials (Chen et al., 2012). However, hydrogen peroxide is aggressive with metal surfaces, so carbon offers a valid alternative as electrode material. Carbon-based electrochemical sensors are commonly fabricated by the screen-printing of carbon inks, which is a suitable approach for mass production of disposable electrodes (Renedo et al., 2007; Wang and Musameh, 2004). Unfortunately, carbon screen-printing requires separate processes and instrumentation with respect to microelectronics and microfabrication. Consequently, it is not pursuable when developing integrated systems. To overcome this problem, it is possible to deposit carbon-based materials on metal electrodes afterwards. Carbon nanotubes (CNTs) are the ideal candidate for their numerous and well-known properties (Cai and Chen, 2004; Gooding et al., 2003). There are several methods to deposit carbon nanotubes on metal electrodes. Electrodeposition is by far one of the most precise and reliable techniques to deposit carbon nanotubes even on sub-mm electrodes (Vashist et al., 2011). Electrodeposition is a method to arrange macromolecules by applying a voltage on an electrode. In the case of carbon nanotubes, they can be dispersed in a biopolymer, like chitosan (CHT), which entraps them in a sort of scaffold thanks to the applied voltage. Chitosan is a natural polysaccharide obtained from the deacetylation of chitin, which can be found in crustaceans, insects, and fungi. Chitosan is largely used in agriculture, in industry, and in medicine for its biocompatibility, antibacterial properties, affinity to proteins, good adhesion, and high permeability to water (Wu et al., 2002). Moreover, CNTs disperse homogeneously in $\mathrm{CHT}$, without loosing their physical and chemical properties. Thanks to the excellent biocompatible properties of CHT, also oxidases can be easily mixed with the CHT/CNTs solution without denaturizing (Qian and Yang, 2006).

In the present paper we will propose a novel approach to monitor metabolites in cell culture media by using a selfcontained platform that we have developed. Conversely to standard procedures that foresee the off-line measurement, we will describe the development of a miniaturized biosensor array to detect glucose and lactate in-situ and on-line. This effort opens the possibility of detecting many others metabolites, thanks to the versatility of the system. The electrochemical-based biosensors were microfabricated with thin film technologies and they consist of several working electrodes sharing a common counter and a common reference electrode. Carbon nanotubes and oxidases were mixed with chitosan and the mixture was electrodeposited on top of the working electrodes. Two different electrodes on the same platform were functionalized with glucose and lactate oxidase, respectively. An ad hoc and low-noise electronics was developed on a printed circuit board to perform the electrochemical measurements and to read the current generated by the electrochemical reaction. An Android application was developed to command the platform and display the metabolite concentration without interfering with the cell culture, while kept in the incubator. Calibration lines and system validation were performed on U937 cell line for both glucose and lactate detection. U937 are hystiocytic lymphoma cells that can differentiate into macrophagelike cells under certain treatments (Ramadan et al., 2013). Many laboratory models investigate the transport of molecules through the epithelial barrier of the gastrointestinal tract. We decided to measure metabolites in these immune cells, since there is a close and strong connection between metabolism and immunoregulation at the gastrointestinal level (Furness et al., 1999). Finally, we also tested the long-term stability of the biosensors and the cross talk among different electrodes, in the perspective to have a more complete metabolic analysis in vitro.

\section{Material and methods}

\subsection{Reagents and chemicals}

Gold Screen-Printed Electrodes (Au-SPEs - model DRP-C220AT) and Multi-Walled Carbon Nanotubes (MWCNTs - diameter $10 \mathrm{~nm}$, length 1-2 $\mu \mathrm{m}, \mathrm{COOH}$ content $5 \%$ ) were purchased from Dropsens (Spain). Chitosan was supplied by Sigma-Aldrich (Switzerland). CHT solution $0.7 \% \mathrm{w} / \mathrm{v}$ was made dissolving CHT flakes in a solution of acetic acid $2 \% \mathrm{pH} 3$ and stirred until complete dissolution. Final $\mathrm{pH}$ was then set to 5. MWCNTs were added at the concentration of $8 \mathrm{mg} / \mathrm{ml}$ and the solution was sonicated for 2-3 h prior to use. Glucose oxidase from Aspergillus Niger (GOD, Grade I, $\geq 300$ units/mg lyophilized) and lactate oxidase from Aerococcus viridans (LOD, Grade II, $\geq 20$ units/mg lyophilized) were purchased from Roche Applied Science (Germany). In the case of enzyme electrodeposition, glucose and lactate oxidase were added to the mix of CHT and MWCNTs in the concentration of $55 \mathrm{mg} / \mathrm{ml}$ and $20 \mathrm{mg} / \mathrm{ml}$, respectively. D-(+)-glucose and lithium L-lactate were purchased from Sigma-Aldrich (Switzerland) in lyophilized powder. Glucose and lactate were dissolved in Phosphate Buffer Saline (PBS) $0.01 \mathrm{M}$ at $\mathrm{pH}$ 7.4.

\subsection{Microfabrication}

Microfabrication was realized at the EPFL Center of MicroNano Technology (CMI). The platform is conceived with gold working electrodes (WEs) placed around a semi-circle, a common gold counter electrode (CE), and a platinum reference electrode (RE). Metal layers are evaporated by physical vapor deposition (PVD) onto $525 \mu \mathrm{m}\langle 100\rangle$ silicon substrate covered by $500 \mathrm{~nm}$ of $\mathrm{SiO}_{2}$. Au and $\mathrm{Pt}$ thin films $(200 \mathrm{~nm})$ are patterned to form the electrodes and connections by photolithography and lift-off processes. A thin film of $\mathrm{Ti}(20 \mathrm{~nm})$ is deposited on top and under the metal layer, as adhesion layer for metals and passivation. All the WEs are identical and they have a diameter of $564 \mu \mathrm{m}$. Connector insulation is achieved by radio-frequency (RF) sputtering of $\mathrm{SiO}_{2}$ and successive wet etching by $\mathrm{BHF}(7: 1)$ of the $\mathrm{SiO}_{2}$ and Ti layers next to the electrodes and pads for electronics connections. The wafer is then diced by using an automatic saw in smaller chips of $9.5 \times 23.3 \mathrm{~mm}^{2}$.

\subsection{Apparatus}

The electrodeposition of CHT/MWCNTs was carried out by using an Autolab electrochemical workstation (N series Potentiostat/Galvanostat, Methrom, Switzerland). The on-line sampling of cell culture media was performed by using a microdialysis probe supplied by Microbiotech AB (Sweden). The probe consists of two coaxial channels, one connected to the inlet and one to the outlet of the probe. The probe is immersed in the culture media and connected to a peristaltic pump (Minipuls 3, Gilson, Switzerland) In the inner channel a perfusate (typically PBS) is flown to a velocity of $13 \mu \mathrm{l} / \mathrm{min}$. When reaching the tip of the probe, the perfusate overflows into the outer channel, which has a membrane directly in contact with the external cell culture media. The cut-off of the membrane is $6 \mathrm{kDa}$, since glucose and lactate have an atomic mass lower than $0.2 \mathrm{kDa}$. The solution leaving the probe is enriched by all the molecules contained in the media with a size smaller than the cut-off of the membrane. The main advantage of this configuration is that the membrane can significantly extend the linear range of the biosensor, playing the role of a diffusion barrier. Moreover, the microdialysis probe can be sterilized and re-used for several times in different cell cultures without contamination issues. The enriched solution is then carried next to the working electrodes. An ad hoc polydimethylsiloxane (PDMS) 


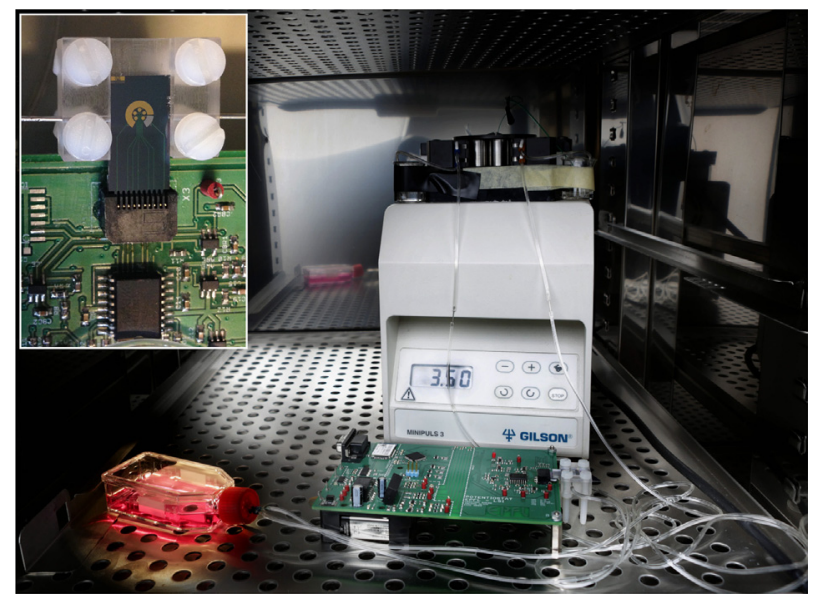

Fig. 1. Overall setup for the on-line monitoring of cell cultures hosted in the incubator. The microdialysis probe is inserted in the cap flask and it is in contact with the cell culture media. The self-contained circuit exchanges data by Bluetooth transmission. The peristaltic pump is used to flow the perfusate and the conditioned media next to the sensing area. Inset: optical photo of the microfabricated platform. The five gold working electrodes are surrounded by the gold counter and the platinum reference electrodes.

chamber described in (Boero et al., 2012) is used to confine the solution on the platform, while Tygon tubes are used to flow the perfusate through the peristaltic pump and waste the solution at the end of the measurement. The electrochemical measurements are carried out by using our developed potentiostat. Fig. 1 shows the overall setup for the on-line monitoring of cell cultures. The microdialysis probe can be fixed to the cap of the flask and immersed in the media. The perfusate is flown by using the peristaltic pump. The potentiostat is battery supplied, so that it can be hosted inside the incubator without wires and cables. The inset of Fig. 1, instead, illustrates the silicon platform of biosensors plugged into our potentiostat. As already mentioned in Section 2.2, the five WEs are arranged in a semi-circle and they are surrounded by the counter electrode. The common reference electrode is the wedge next to the counter.

Images of electrodeposited MWCNTs are acquired by using a Philips/FEI XL-30 F (Netherlands) Scanning Electron Microscope (SEM). The resolution in UHR mode is $2.5 \mathrm{~nm}$ at $1 \mathrm{kV}$. Pictures of the magnified working electrodes are captured by a Nikon Eclipse LV100 optical microscope equipped with a digital photo camera Camedia C-5060.

\subsection{Nanostructuration by electrodeposition}

For the electrodeposition of CHT and MWCNTs, the working electrode of the Au-SPE is used as counter electrode. The SPE is placed close and opposite to the working electrodes of the platform, in order to collect as much current as possible. The reference and the working electrodes, instead, belong to the microfabricated platform. A manual switch as connector is used to change among the five working electrodes. The CHT/MWCNTs solution is sonicated for $10 \mathrm{~min}$ and vortex before using it. A volume of around $100 \mu \mathrm{l}$ is drop cast on top of the microfabricated electrodes, in order to cover all of them. Then, the Au-SPE is moved close to the platform and kept in contact with the CHT/MWCNTs solution. The applied voltage and the deposition time are optimized in order to guarantee the polymerization of the chitosan, as well as avoiding electrode burning. Voltage values found in the literature regarding gold electrodes report values around 2-3 V (Wu et al., 2007). However, microfabricated electrodes are thin (thickness $200 \mathrm{~nm}$ ) and small (diameter $564 \mu \mathrm{m}$ ). If the current density is too high, there is the risk of electrode delamination or burning. The best results in terms of homogeneity and MWCNTs density are obtained with an applied voltage of $1.2 \mathrm{~V}$ for an electrodeposition time of $30 \mathrm{~min}$. In the case of CHT/MWCNTs and enzyme mixture, the electrodeposition is applied with the same parameters.

\subsection{Evaluation of the standard deviation}

Data analysis and the performance criteria of the biosensor (sensitivity and detection limit) are calculated according to the guide given by IUPAC (Thévenot et al., 1999). For the computation of the standard deviation on sensitivity and on limit of detection, we followed the theory of errors. Regarding the sensitivity, standard deviation of the slope $\delta_{\text {Slope }}$ is calculated as

$\delta_{\text {Slope }}=\frac{\delta_{y / x}}{\sqrt{\left(x_{i}-\bar{x}\right)^{2}}}$

where $x_{i}$ is the value of each concentration and $\bar{x}$ is the mean of all concentration injected. $\delta_{y / x}$ is defined as the standard error

$\delta_{y / x}=\frac{\sqrt{\Sigma\left(y_{i}-\hat{y}\right)^{2}}}{(n-2)}$

where $\mathrm{y}_{i}$ is the mean current value for each concentration in a certain interval time, when current reaches the steady-state, $\hat{y}$ is the corresponding value on the regression line, and $n$ is the number of calibration points, including the blank signal. Therefore, the standard error of the sensitivity $\delta_{S}$ is defined as

$\delta_{S}=t_{n-2} \cdot \delta_{\text {Slope }}$

where $t_{n-2}$ is the t-distribution with $n-2$ degrees of freedom, and $\delta_{S}$ is the sensitivity error referred to a confidential interval of $95 \%$.

The standard error of the $y_{\text {intercept }}$ is calculated as follows:

$\delta_{\text {int ercept }}=\delta_{y / x} \cdot \sqrt{\frac{\sum x_{i}^{2}}{n \cdot \sum\left(x_{i}-\bar{x}\right)^{2}}}$

following IUPAC definition, the limit of detection $(L O D)$ is defined as follows:

$L O D=3 \cdot \frac{\delta_{\text {blank }}}{S}$

where $\delta_{\text {blank }}$ is the standard error referred to the blank signal and $S$ is the sensitivity. Therefore, we need several measurements of the blank signal. Then, we decided to divide the acquired current of the blank signal, recorded typically for several minutes, in three intervals of $20 \mathrm{~s}$ and to calculate the standard deviation of each sample stream. Then, we computed the mean of the three standard deviations, obtaining the value of $\delta_{\text {blank }}$. The standard error on the limit of detection, is calculated as:

$\delta_{\text {LOD }}=\left(\frac{\delta_{S}}{S}+\frac{\delta_{\text {blank }}}{\overline{\text { blank }}}\right) \cdot L O D$

\subsection{Cell culture}

U937 cells are a suspension line isolated from the histiocytic lymphoma of a 37-year-old male, which is often used to study the behavior of monocytes. Proliferating cells are maintained in RPMI1640 media with stable glutamine (PAA, Switzerland), supplemented with $10 \%$ Newborn Calf Serum (NCS, Sigma-Aldrich, USA origin) in $25 \mathrm{~cm}^{2}$ culture flasks (Cellstar ${ }^{\circledR}$, Greiner, Germany), in a $5 \% \mathrm{CO}_{2}$ atmosphere at $37^{\circ} \mathrm{C}$. This cell line duplicates every $24 \mathrm{~h}$ and its ideal density is $7.5 \times 10^{5}$ cells $/ \mathrm{ml}$. From the original density, cells were cultivated until their density resulted around $7.5 \times 10^{5}$ cell $/ \mathrm{ml}$. Then, this volume was divided in 4 different flasks, so that the ideal density was reached every two days. For dividing and seeding, U937 are centrifuged at $800 \mathrm{rpm}$ for $5 \mathrm{~min}$ in order to 
obtain the pellet deposition. Then, old medium is collected and replaced with fresh one. Finally, the pellet is re-suspended in the new medium and transferred to a new flask with the wished density rate. Medium in the stock flasks was changed at the beginning of the monitoring before cell proliferation. Cells are seeded at three different densities: $1.2 \times 10^{5}, 1.8 \times 10^{5}, 3.0 \times 10^{5}$ cell $/ \mathrm{ml}$. Surnatant conditioned medium is collected for glucose and lactate level measurements at 16, 40,64, and $88 \mathrm{~h}$ after cell seeding.

\subsection{MTT assay}

Cell proliferation is studied by MTT biochemical approach for every collection of surnatant. MTT (3-[4,5-dimethylthiazol-2-yl]2,5-diphenyl tetrazolium bromide) produces a yellowish solution that is converted to dark blue, water-insoluble MTT formazan by mitochondrial dehydrogenases of living cells. A volume of $10 \mu \mathrm{l}$ of cell suspension with conditioned surnatant is mixed with $10 \mu \mathrm{l}$ of MTT. A volume of $10 \mu \mathrm{l}$ mixture is then injected in the two parts of a Burker chamber and cell are counted with the use of a microscope. Viable cells appear white and bright, while dead cells are darker. The number of viable cells is averaged between the two chambers, doubled (because the dilution rate of MTT is 1:2) and multiplied by $10^{5}$, in order to obtain the final density of the cells for each $\mathrm{ml}$ of volume.

\section{Results and discussion}

\subsection{Dedicated electronics for the biosensor}

A custom potentiostat was designed to monitor the current flowing through the cell in real-time and to return its value to portable devices, such as smartphones and tablets. The potentiostat is achieved with off-the-shelf components, accurately selected to have low current leakage. The schematic of the sensor is reported in Fig. 2A. The electrochemical cell is driven by applying a constant voltage $V_{O X}$ between the reference electrode and one of the five working electrodes. The voltage $V_{R E}$ at the reference electrode is acquired by using a voltage buffer; thus, no current flows on that electrode. This is important to avoid the polarization of the electrode, which can cause some changes in the interfacial potential difference. Moreover, the polarization of the reference electrode can cause some material depletion due to the current flowing through it (Ahmadi and Jullien, 2008). The constant voltage $V_{O X}$ is then summed to $V_{R E}$ and the resulting voltage $V_{R E}+V_{O X}$ is applied to the working electrode selected by the user. In order to protect the cell, $V_{R E}$ is constantly monitored by the microcontroller to disconnect the voltage driver while the reference electrode is floating. This feature prevents damages to the electrodes when the solution on top of the electrochemical cell dries or the user makes some errors. If the reference electrode is floating, the circuit does not force too much current density on the electrode.

The current flowing on the counter electrode is read by the block current sensing (refer to Fig. 2A). That block performs a current-to-voltage conversion. The relation between the input current $I_{C E}$ and the output voltage $V_{O U T}$ is as follows:

$H(s)=\frac{V_{\text {OUT }}(s)}{I(s)}=-\frac{R_{1} R_{2}+R_{f} R_{2}+R_{f} R_{1}+s C R_{1} R_{2} R_{f}}{R_{1}\left(1+s C R_{f}\right)}$.

The transfer function of the block is reported in Fig. 2B. The lowpass behavior has been designed to efficiently detect the DC current. In that case, the transfer function can be expressed as

$\left.H(s)\right|_{S-0}=\frac{V_{\text {OUT }}}{I} .=-\frac{R_{1} R_{2}+R_{f} R_{2}+R_{f} R_{1}}{R_{1}}$

The voltage $V_{\text {OUT }}$ is then digitized and stored in a microcontroller. These values are sent to portable devices by using a Bluetooth Serial Port Protocol (SPP). The system is designed to read currents up to $185 \mathrm{nA}$ with a current resolution of $25 \mathrm{pA}$.

Passive and active guard rings are used to protect the sensitive paths, such as RE, CE, and the working electrodes. Passive guard rings involve the interruption of the solder resist of the sensitive paths against the surrounding paths. Active rings involve physical metal rings surrounding the sensitive path and driven to the same voltage as the sensitive path by means of voltage buffers. The system is powered with two $9 \mathrm{~V}$ batteries in series. Different voltages are generated on board for the analog and the digital parts. PI-filters are used to remove noise from the power supplies.

\subsection{Android application}

We developed a dedicated Android application to connect the electrochemical platform with portable devices. This solution supports the self-contained and in-situ device that can be placed inside the incubator, so that every time the user wants to get information from the cell culture, he/she does not need to open the incubator or extract the media. Indeed, the minimization of cell culture manipulation decreases the risk of culture contamination and interference with the biological processes.

BlueCells is an Android application designed to connect the electrochemical sensor depicted in the previous section with portable devices, such as smartphones and tablets. The application is connected to the sensor via Bluetooth using a SPP communication profile. In Fig. $2 \mathrm{C}$ is shown a screenshot of the application. The user can set the sampling instants $t_{1}$ and $t_{2}$, the sampling period, and the working electrode to be used among the available ones. Furthermore, a moving average filter can be applied to the samples returned by the biosensor. After data acquisition, the user can analyze the whole signal by scrolling and zooming it directly on the device screen. This feature is enabled by using an adaptive downsampling algorithm. Finally, the acquired data can be saved into an external storage device in text format and eventually sent by email.

The instruction set of the communication protocol consists of four main commands, each having a length of two bytes. The three most significant bits of every instruction define the command type. The protocol is designed so that the sensor is not aware of the communication status, but simply satisfies every new request from the application. It reduces the complexity on the sensor side, enabling low-power applications.

\subsection{Characterization of the modified gold working electrodes}

Electrodeposition was chosen as a strategy for the selective immobilization of both MWCNTs and enzymes on the working electrodes of the platform. Indeed, this technique enables to succeed in a homogeneous deposition of carbon nanotubes, and the preservation of the functional and electrochemical properties of both MWCNTs and enzymes. Moreover, electrodeposition is highly selective and specific. Fig. 3A shows a schematic of the CHT/ MWCNTs and enzymes arrangement on the surface of the electrode. At low pH, most of the primary amino-groups composing chitosan are protonated and positively charged, making it soluble in aqueous solution. At $\mathrm{pH}$ higher than $\mathrm{p} K_{a}$ (the acid dissociation constant), chitosan becomes insoluble forming a hydrogel (Park et al., 2006). CHT flakes and MWCNTs are dissolved in a solution at 

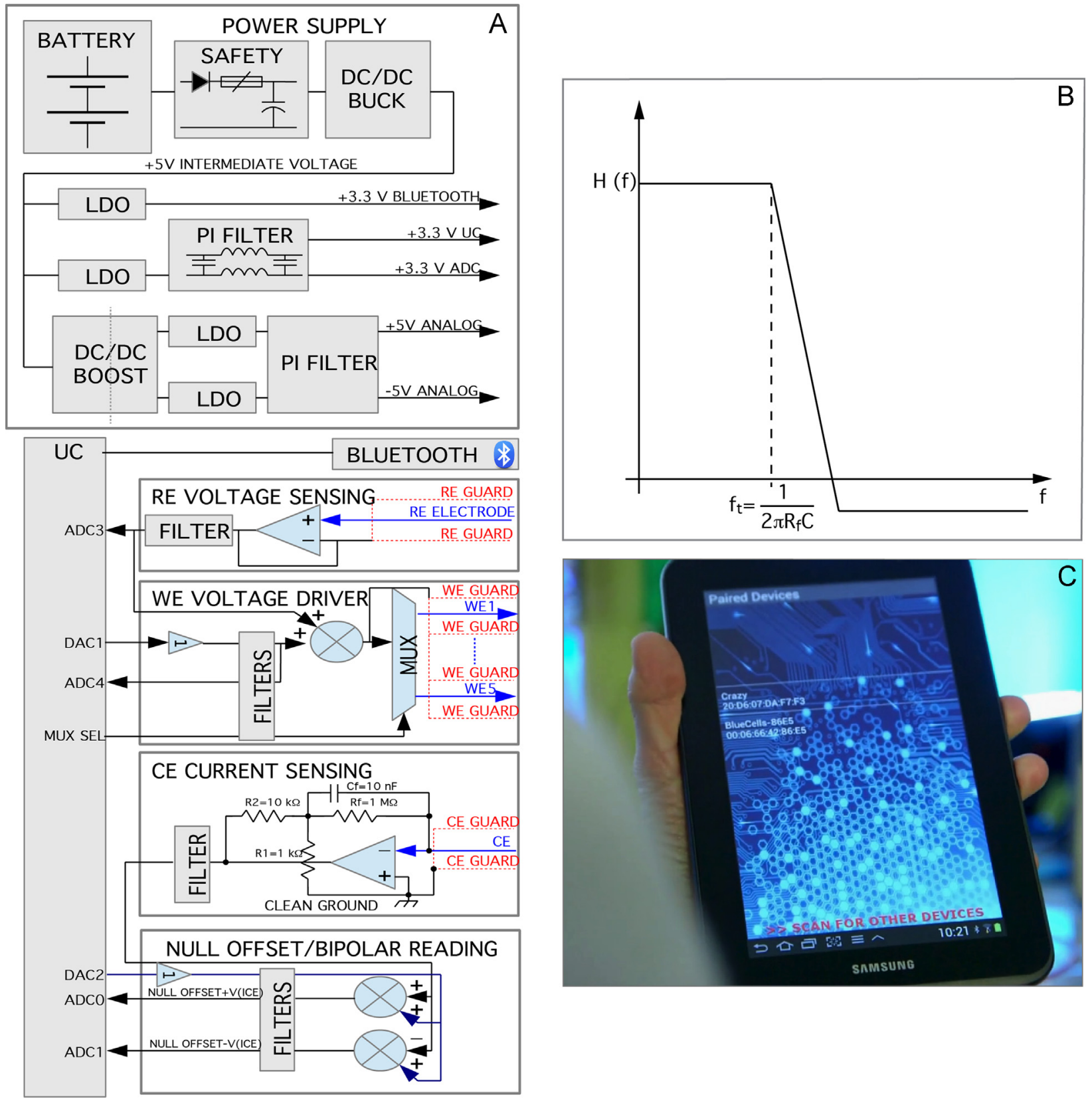

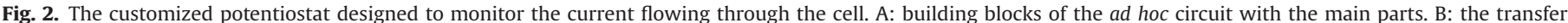
function related to the block "current sensing". C: a screenshot of the Android application "BlueCells" running on a tablet.

$\mathrm{pH}$ 3, so that chitosan is water-soluble. Then, $100 \mu \mathrm{l}$ of that solution are used to cover the five working electrodes. By exploiting the intrinsic properties of chitosan, the polarization of one particular electrode can create a localized region of higher $\mathrm{pH}$ that can exceed chitosan $\mathrm{p} K_{a}$, forcing the local CHT assembly and the entrapment of any other compound present in the original solution, such as the carbon nanotubes and the enzymes in our case. Fig. $3 \mathrm{~B}$ and $\mathrm{C}$ shows a comparison before and after the electrodeposition of CHT on the gold working electrode. CHT/MWCNTs mix results to be homogeneous and confined within the electrode area at the macro-scale. At the micro-scale, the electro-polymerized CHT appears as a spongy scaffold mixed with disorganized single and bundles MWCNTs, as shown in Fig. 3E. Thanks to the porosity of the polymerized CHT, small molecules like glucose and lactate can access the nanostructures and the enzymes, while bigger molecules potentially interfering with the measurements are blocked. The evidences of the successful electro-polymerization are visible if comparing Fig. 3E with Fig. 3D. Indeed, the bare electrode shows particle aggregates on the rough flat surface (see Fig. 3D). On the contrary, once the polymer is assembled, it forms a tridimensional structure, as shown in Fig. 3E.

\subsection{Biosensor calibration for glucose and lactate in PBS and in media}

Measurements related to the calibration of the biosensors require different experimental setups when performed in PBS or in the cell culture media. In the case of PBS, no microdialysis probe is used for the measurements. Nominal concentrations from 0 up to $1 \mathrm{mM}$ with steps of $200 \mu \mathrm{M}$ of glucose and lactate are prepared and exchanged every $5 \mathrm{~min}$ at the inlet and let flow in the fluidic 

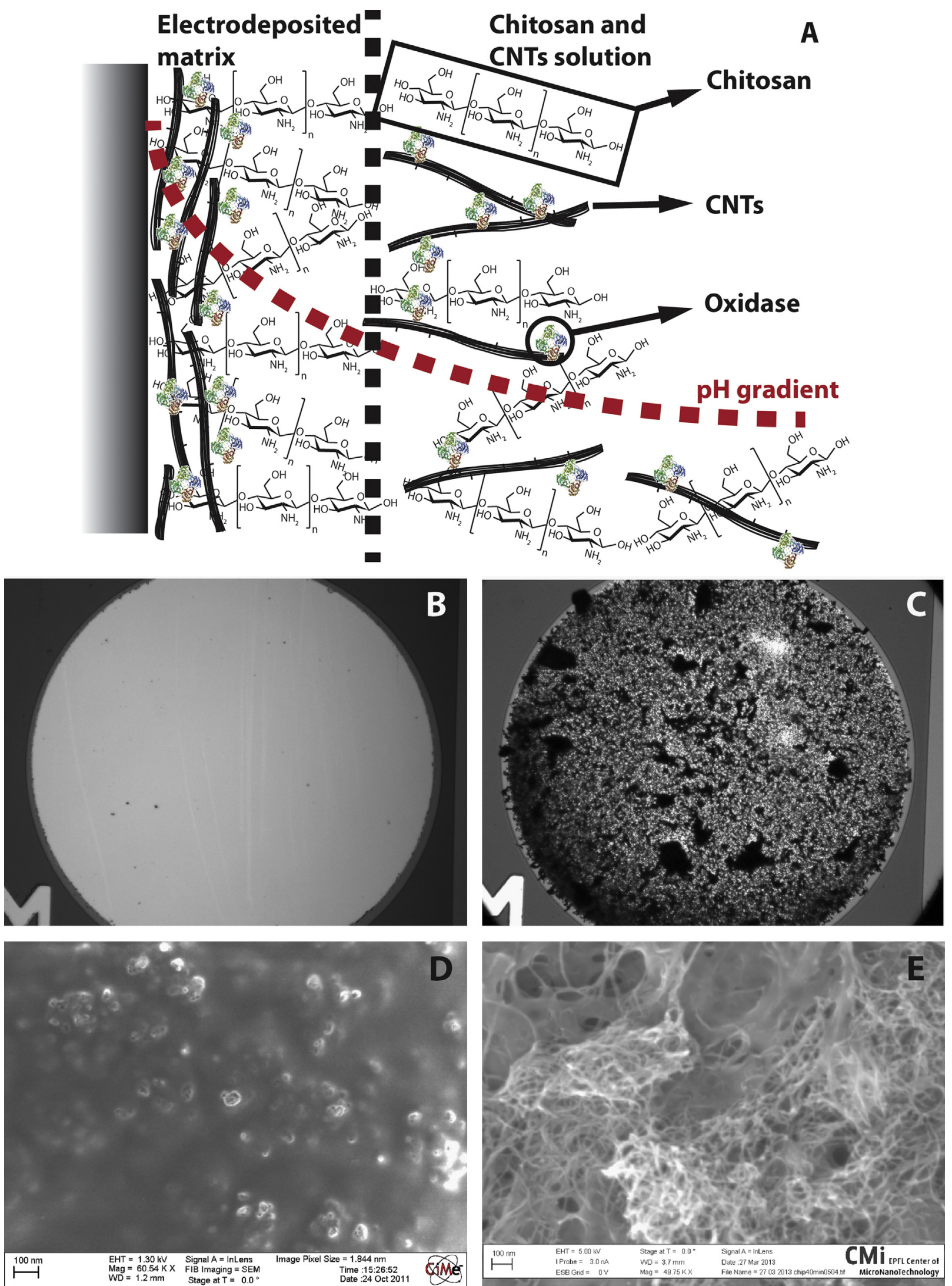

Fig. 3. Selective immobilization of the CHT/MWCNTs/enzyme mixture on top of a particular working electrode. A: a schematic of the CHT/MWCNTs/enzyme arrangement on the surface of the electrode. B and D: optical and SEM images of the bare working electrode. C and E: optical and SEM images of the working electrode after the electrodeposition. 
system. The flow is set to $13 \mu \mathrm{l} / \mathrm{min}$ and the calibrations always start with PBS flowing through the chamber. The initial current value related to PBS is considered as the baseline, so that the successive values of current are referred to the baseline value. Glucose biosensors showed a sensitivity of $410 \pm 50 \mathrm{nA} \mathrm{mM}{ }^{-1} \mathrm{~mm}^{-2}$ and a detection limit of $14 \mu \mathrm{M}(\mathrm{S} / \mathrm{N}=3 \sigma)$, while for lactate biosensors the sensitivity is of $420 \pm 90 \mathrm{nA} \mathrm{mM}^{-1} \mathrm{~mm}^{-2}$ and the detection limit of $1 \mu \mathrm{M}(\mathrm{S} / \mathrm{N}=3 \sigma)$.

In the case of cell culture media, glucose is dissolved in different concentrations in D5030 (by Sigma-Aldrich), which is a glucose-free media, to perform the biosensor calibration, while lactate is dissolved in the one also used for the cell cultures. Both the metabolites are tested within a range from 0 to $25 \mathrm{mM}$ with steps of $5 \mathrm{mM}$. The concentrations for both metabolites are chosen in the $\mathrm{mM}$ range accordingly to previous data measured in the literature for the case of murine embryonic cells (Hwang et al., 2009) and NG-108 neural cells (Leegsma-Vogt et al., 2004). Moreover, the initial concentration of glucose in RPMI-1640 is $11 \mathrm{mM}$, according to the datasheet provided by PAA, which is in the middle of the tested concentration window. In this case the microdialysis probe is immersed in the media and flasks with different metabolite concentrations are exchanged at the inlet of the fluidic system. Glucose biosensors showed a sensitivity of $4.7 \pm 1.3 \mathrm{nA} \mathrm{mM}{ }^{-1} \mathrm{~mm}^{-2}$ and a detection limit of $1.4 \mathrm{mM}$ $(\mathrm{S} / \mathrm{N}=3 \sigma)$, while for lactate biosensors the sensitivity is of $12.2 \pm$ $3.8 \mathrm{nA} \mathrm{mM}^{-1} \mathrm{~mm}^{-2}$ and a detection limit of $0.3 \mathrm{mM}(\mathrm{S} / \mathrm{N}=3 \sigma)$. By comparing the sensitivity values for calibration in PBS and in media it is possible to notice that there is a difference of about two orders of magnitude. The microdialysis probe introduces a clear dilution effect that makes the biosensors less sensitive in cell culture media with respect to detection in PBS. On the other side, it enables to enlarge the concentration window to achieve metabolite monitoring in the physiological range for cell cultures.

\subsection{Glucose and lactate detection in cell culture media}

Biosensor calibration is used both to check the window range of linearity and to determine the sensitivity for the biosensor. For measurements performed in the cell culture media, a two-point calibration is carried out before the measurement of the surnatant. For glucose the two known concentrations are $2 \mathrm{mM}$ and $11 \mathrm{mM}$, while for lactate they are $0 \mathrm{mM}$ and $20 \mathrm{mM}$ in RPMI-1640. Therefore, the measurement protocol is the following: the blank concentration, the highest known concentration, the blank concentration, the surnatant, the highest known concentration, and finally the blank concentration. The media is measured every 16 , 40,64 , and $88 \mathrm{~h}$ for the three cell densities. We also counted the related cell viability and we checked the $\mathrm{pH}$ of the media for each time-point. During $88 \mathrm{~h}$ of cultivation, we were expecting an increase in cell density, so that both glucose and lactate concentrations would be affected.

Figs. 4 and 5 show glucose uptake and lactate production of U937 cell line for densities of $1.2 \times 10^{5}, 1.8 \times 10^{5}$, and $3.0 \times 10^{5}$ cell $/ \mathrm{ml}$ (density values are related to the moment of cell seeding). According to our expectations, glucose consumption is higher for higher cell density and it is more evident in the case of the highest concentration. In the latter case, indeed, glucose availability is almost at $50 \%$ after $40 \mathrm{~h}$ of cultivation. For cell density of $1.2 \times 10^{5}$ cell $/ \mathrm{ml}$, instead, it is evident a slower metabolism and a glucose consumption that remains roughly constant for all the time of cultivation. On the other hand, lactate begins to be produced when glucose concentration goes below roughly 8-9 $\mathrm{mM}$ and cells start suffering for lack of nutrient. Indeed, for all the three densities, lactate production has two different rates before and after $40 \mathrm{~h}$ of cultivation. Lactate trend after $40 \mathrm{~h}$ have much higher slope with respect to the trends related to the beginning of the cultivation.

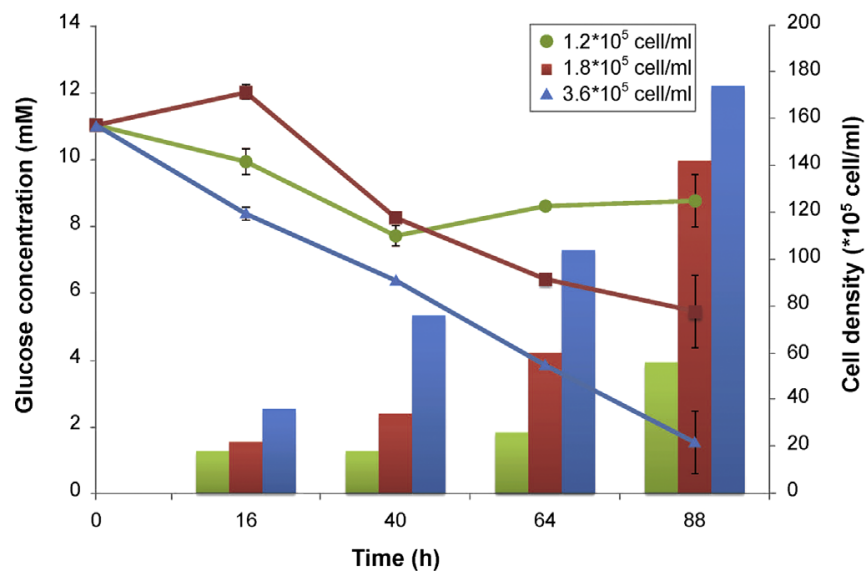

Fig. 4. Glucose uptake of the U937 cell line over $88 \mathrm{~h}$. The green line is related to the lowest cell density $\left(18 \times 10^{5} \mathrm{cell} / \mathrm{ml}\right)$, the red line is related to the middle cell density $\left(22 \times 10^{5} \mathrm{cell} / \mathrm{ml}\right)$, and the blue line is related to the highest cell density $\left(36 \times 10^{5} \mathrm{cell} / \mathrm{ml}\right)$. The bars, instead, are related to the cell viability: green, red, and blue bars are related to $18 \times 10^{5} \mathrm{cell} / \mathrm{ml}, 22 \times 10^{5} \mathrm{cell} /{ }^{-1}$, and $36 \times 10^{5} \mathrm{cell} / \mathrm{ml}$, respectively. (For interpretation of the references to color in this figure legend, the reader is referred to the web version of this article.)

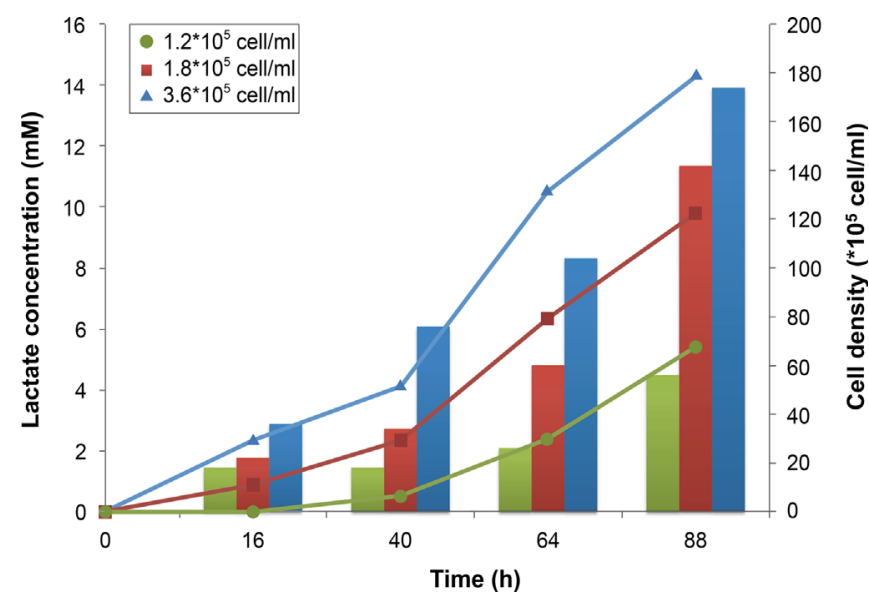

Fig. 5. Lactate production of the U937 cell line over $88 \mathrm{~h}$. The green line is related to the lowest cell density $\left(18 \times 10^{5} \mathrm{cell} / \mathrm{ml}\right)$, the red line is related to the middle cell density $\left(22 \times 10^{5}\right.$ cell $\left./ \mathrm{ml}\right)$, and the blue line is related to the highest cell density $\left(36 \times 10^{5} \mathrm{cell} / \mathrm{ml}\right)$. The bars, instead, are related to the cell viability: green, red, and blue bars are related to $18 \times 10^{5} \mathrm{cell} / \mathrm{ml}, 22 \times 10^{5} \mathrm{cell} / \mathrm{ml}$, and $36 \times 10^{5} \mathrm{cell} / \mathrm{ml}$, respectively. (For interpretation of the references to color in this figure legend, the reader is referred to the web version of this article.)

That is because when cells are approaching the ideal concentration of $7.5 \mathrm{cell} / \mathrm{ml}$, they will optimally reproduce and doubling their density every $24 \mathrm{~h}$.

\subsection{Long-term stability of the biosensor}

Another aspect to consider when characterizing a new type of functionalization is the long-term stability of the biosensors. We estimate the enzyme functionality when entrapped in a matrix of chitosan and MWCNTs by considering lactate oxidase as an example. The same functionalized electrode is tested over 20 days and its sensitivity is then evaluated in function of the time. The measurements are performed after 1, 2, 3, 5, and 10 days in PBS pH 7.4 at the applied potential of 0.65 vs. $\mathrm{Ag} / \mathrm{AgCl}$. After each experiment, the platform is washed with PBS and stored in the fridge when not in use. Sensitivities are normalized with respect to the value acquired the first day $\left(450 \mathrm{nA} \mathrm{mM}^{-1} \mathrm{~mm}^{-2}\right)$ and the retained activity of the biosensor is calculated. Measurements show a stable behavior of the biosensor for the first 10 days with 


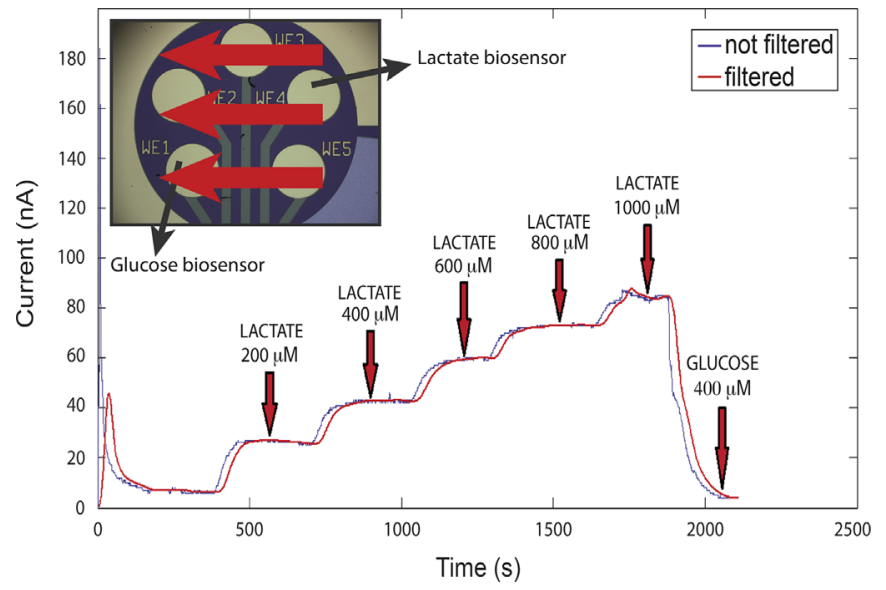

Fig. 6. Chronoamperometry related to the lactate detection. Steps of $200 \mu \mathrm{M}$ of lactate are successively injected in the system. The last injection, instead, is done with $400 \mu \mathrm{M}$ of glucose to check the cross talk among the working electrodes. Inset: optical image of the five WEs and their geometrical disposition related to the flow. WE1 is functionalized with glucose oxidase, while WE4 is functionalized with lactate oxidase.

a retained activity higher then $80 \%$ (data not shown) and within $60 \%$ by 20 days. Indeed, the biosensor sensitivity varies between 380 and $450 \mathrm{nA} \mathrm{mM}{ }^{-1} \mathrm{~mm}^{-2}$ within the first 10 days. The obtained results are in agreement with previous works based on immobilization of oxidases in chitosan (Wu et al., 2009; Tsai et al., 2007; Tangkuaram et al., 2007).

\subsection{Evaluation of the cross talk among the working electrodes}

The advantage of using electrodeposition is the possibility of selectively functionalizing each working electrode with a different oxidase by applying a fixed potential just to the working electrode of interest. Since the platform is functionalized with different enzymes belonging to the family of oxidases, and all of them produce $\mathrm{H}_{2} \mathrm{O}_{2}$ as by-product of the enzymatic reaction, the investigation of the interference among the different oxidases is fundamental also in relation with the electrode geometry. Indeed, it is important to distinguish the contribution of the enzyme under measurement from the contribution of the others. Moreover, since the system is conceived under flow, we also want to explore the best geometry of the electrodes relatively to flow direction to minimize the effect of the cross talk.

Two working electrode are functionalized with lactate oxidase and glucose oxidase, respectively, as depicted in the inset of Fig. 6. The two WEs are misaligned with respect to the direction of the flow (red arrows in the figure). The detection is performed on the electrode functionalized with lactate oxidase, upstream with respect to the flow. Lactate is diluted in PBS and successively injected in higher concentrations for each step (200 $\mu$ M lactate per step). The last injection, instead, is performed with $400 \mu \mathrm{M}$ of glucose, while monitoring the lactate biosensor. We are interested in investigating and minimizing the cross talk due to redox reaction generated at the glucose biosensor site on the measurement carried out upstream, i.e. at the lactate biosensor. Fig. 6 shows current steps recorded at the lactate biosensor due to lactate injection and the absence of cross talk when injecting glucose in the fluidic cell. We can definitely assert that the flow ensures to bring all the by-products of the redox reaction away from the platform chamber. From this test it is possible to conclude that the presence of glucose does not interfere with the measurement of lactate. On the contrary, if the working electrodes are aligned and parallel to the flow, $\mathrm{H}_{2} \mathrm{O}_{2}$ produced by the upstream electrode will interfere with the measurement of the downstream electrode.

\section{Conclusion}

The present research is focused on the development of a selfcontained device for metabolite detection, in particular glucose and lactate, in cell culture media. Conversely to standard procedures, this approach succeeds in the on-line and in-situ measurement of different metabolites on the same platform. We exploited electrodeposition as a mean to precisely deposit carbon nanotubes and oxidases on top of metal electrodes. By taking advantage of the intrinsic properties of chitosan, the polarization of an electrode can create a localized region of higher $\mathrm{pH}$ which forces the local CHT polymerization and the entrapment of any other compound present in the original solution. This technique enables us to succeed in a homogeneous deposition of carbon nanotubes, and the preservation of the functional and electrochemical properties of both MWCNTs and enzymes. We also developed an ad hoc electronics for both applying a constant voltage and reading out the current of the electrochemical cell. The circuit was developed on a printed circuit board and it is self-contained, powered by two $9 \mathrm{~V}$ batteries. An Android application was developed to interface the circuit with smartphones and tablets. The communication is based on Bluetooth SPP protocol, in order to be as much versatile as possible. Glucose and lactate biosensors were characterized in terms of sensitivity and limit of detection. Then, the device was validated in U937 cell line for the detection of metabolites over $88 \mathrm{~h}$. The measured glucose and lactate trends are fully consistent with the viability and density of the cells. Finally, long-term stability of the lactate biosensor is evaluated for 20 days. The retained activity of the biosensor is within $80 \%$ by the first 10 days and within $60 \%$ by 20 days. The design and development of a device such as this one pave the way to novel and more effective opportunities to improve pharmaceutical manufacturing, quality assurance, and process optimization through process control and analysis.

\section{Acknowledgments}

The authors would like to thank all the CMI staff from EPFL for their support on microfabrication processes. C. Baj-Rossi, I. Taurino, and A. Cavallini are acknowledged for useful discussion on electrodeposition by using chitosan. CIME staff is acknowledged for thei help in acquiring SEM images. Financial support is partially from the NanoTera ch project I-Needle, and partially from the NanoSys project, within the program ERC-2009-AdG-246810.

\section{References}

Ahmadi, M.M., Jullien, G.A., 2008. In: Iniewski, K. (Ed.), VLSI Circuits for Biomedical Applications. Artech House, Boston/London, pp. 265-285.

Boero, C., Olivo, J., De Micheli, G., Carrara, S., 2012. IEEE Trans. Biomed. Circuits Syst. $6,479-485$.

Cai, C., Chen, J., 2004. Anal. Biochem. 332, 75-83.

Carrara, S., Cavallini, A., Erokhin, V., De Micheli, G., 2011. Biosens. Bioelectron. 26 3914-3919.

Chen, W., Cai, S., Ren, Q.Q., Wen, W., Zhao, Y.D., 2012. Analyst 137, 49-58.

D’Orazio, P., 2011. Clin. Chim. Acta 412, 1749-1761.

FDA Guidelines, 2004. Fed. Regist. 69, 59257-59258.

Furness, J.B., Kunze, W.A.A., Clerc, N., 1999. Am. J. Physiol. Gastrointest. Liver Physiol. 277, 922-928.

Gooding, J.J., Wibowo, R., Liu, J., Yang, W., Losic, D., Orbons, S., Mearns, F.J., Shapter J.G., Hibbert, D.B., 2003. J. Am. Chem. Soc. 125, 9006-9007.

Hwang, Y.S., Cho, J., Tay, F., Heng, J.Y.Y., Ho, R., Kazarian, S.G., Williams, D.R. Boccaccini, A.R., Polak, J.M., Mantalaris, A., 2009. Biomaterials 30, 499-507.

Jacobs, C.B., Peairs, M.J., Venton, B.J., 2010. Anal. Chim. Acta 662, 105-127. 
Kimmel, D.W., LeBlanc, G., Meschievitz, M.E., Cliffel, D.E., 2012. Anal. Chem. 84, 685-707.

Leegsma-Vogt, G., Venema, K., Brouwer, N., Gramsbergen, J.B., Copray, S., Korf, J., 2004. Anal. Chem. 76, 5431-5435.

Park, J.J., Luo, X., Yi, H., Valentine, T.M., Payne, G.F., Bentley, W.E., Ghodssi, R. Rubloff, G.W., 2006. Lab Chip 6, 1315-1321.

Qian, L., Yang, X., 2006. Talanta 68, 721-727.

Ramadan, Q., Jafarpoorchekab, H., Huang, C., Silacci, P., Carrara, S., Koklu, G., Ghaye, J., Ramsden, J., Ruffert, C., Vergeres, G., Gijs, M.A.M., 2013. Lab Chip 13, 196-203.

Renedo, O.D., Alonso-Lomillo, M.A., Arcos Martínez, M.J., 2007. Talanta 73, 202-219.

Tangkuaram, T., Ponchio, C., Kangkasomboon, T., Katikawong, P., Veerasai, W., 2007. Biosens. Bioelectron. 22, 2071-2078.

Temiz, Y., Kilchenmann, S., Leblebici, Y., Guiducci, C., 2011. Electron. Lett. 47, 22-24.
Thévenot, D.R., Toth, K., Durst, R.A., Wilson, G.S., 1999. Pure Appl. Chem. 71, 2333-2348.

Tsai, Y.C., Chen, S.Y., Liaw, H.W., 2007. Sens. Actuators B 125, 474-481.

Vashist, S.K., Zheng, D., Al-Rubeaan, K., Luong, J.H.T., Sheu, F.S., 2011. Biotechnol. Adv. 29, 169-188.

Vojinović, V., Cabral, J.M.S., Fonseca, L.P., 2006. Sens. Actuators B 114, 1083-1091.

Wang, J., Musameh, M., 2004. Analyst 129, 1-2.

Wu, Z., Feng, W., Feng, Y., Liu, Q., Xu, X., Sekino, T., Fujii, A., Ozaki, M., 2007. Carbon 45, 1212-1218.

Wu, L.Q., Gadre, A.P., Yi, H., Kastantin, M.J., Rubloff, G.W., Bentley, W.E., Payne, G.F., Ghodssi, R., 2002. Langmuir 18, 8620-8625.

Wu, H., Wang, J., Kang, X., Wang, C., Wang, D., Liu, J., Aksay, I.A., Lin, Y., 2009. Talanta 80, 403-406. 